Ronald Jefferson Martins ${ }^{1}$

Cléa Adas Saliba Garbin ${ }^{2}$

ArtênioJosé Ísper Garbin ${ }^{2}$

Suzely Adas Saliba Moimaz ${ }^{3}$

\section{Absenteísmo por motivos odontológico e médico nos serviços público e privado}

\section{Absenteeism in public and private jobs due to dental or medical problems}

\begin{abstract}
Resumo
O absenteísmo é um assunto de interesse crescente devido ao atual contexto econômico de competitividade, que faz com que as empresas procurem meios para diminuir sua ocorrência, aumentando a rentabilidade e com isso crescendo de forma sustentada. Procurou-se estudar o absenteísmo por razões odontológicas e médicas, nos serviços público e privado, analisando se a idade, o sexo e a função do trabalhador, além do regime empregatício, interferem na sua ocorrência. Desenvolveu-se o estudo na prefeitura do município de Araçatuba, São Paulo, Brasil, e em uma indústria acrílica. Para a coleta dos dados, analisaram-se todos os atestados odontológicos e médicos, que deram entrada nas empresas no período de janeiro a junho de 2002 e as listagens dos trabalhadores. Verificou-se que a falta por motivos odontológicos tiveram pouco peso sobre o total de faltas por motivo de doença, além de provocarem o afastamento do trabalhador por um período menor. As variáveis idade, sexo, função e regime empregatício influenciaram na ocorrência do absenteísmo ao trabalho. A disponibilidade dessas informações deverá ser muito importante, pois, visto as inúmeras variáveis envolvidas, facilitarão a tomada de decisões das distintas estratégias para sua prevenção.
\end{abstract}

Palavras-chave: absenteísmo, saúde ocupacional, saúde bucal, setores público e privado.

\begin{abstract}
Absenteeism is a subject of increasing interest. Due to the current economical context based on competition, and in order to profit and grow in a sustainable way, companies have been trying to find ways of decreasing its rates. The purpose of this study was to analyze how much the worker's age, genre, occupation as well as the kind of management he is submitted to, could affect the incidence of absenteeism caused by medical or dentistry problems in public and private services. The research was held at an acrylic industry in Araçatuba, a town in the state of São Paulo, Brazil. Data collecting consisted of analyzing the doctors' and dentists' certificates workers handed in to their employers, to explain their absences from work, from January to June 2002. The results showed that absences caused by dental problems were much rarer and for a shorter period than the absences caused by medical problems. The workers' age, genre, occupation as well as the kind of management they were submitted to, influenced the incidence of their absences from work. Making these information available is of great importance. Their different aspects will help choosing the correct strategy to decrease absenteeism rates.
\end{abstract}

Keywords: absenteeism, occupational health, oral health, public and private sector. 


\section{Introdução}

Duas razões levam à implantação de serviços de saúde especificamente para o grupo de trabalhadores: a melhora ou a manutenção das condições de saúde e o controle do absenteísmo (Pinto, 1992).

A palavra "absenteísmo" era aplicada aos proprietários rurais que abandonavam o campo e tendiam a viver nas cidades, sendo que, com o advento da revolução industrial, o termo passou a ser aplicado aos trabalhadores com tendência a faltar ao serviço (Rocha, 1981).

No atual contexto econômico de competitividade, o absenteísmoé um motivo de interesse crescente, pois quanto menor for sua ocorrência, maior será a capacidade das empresas de aumentarem sua rentabilidade e conseguirem um crescimento sustentado (Muñoz, 1997).

Midorikawa (2000) descreve dois tipos de absenteísmo: o absenteísmo pela falta ao trabalho e o absenteísmo de "corpo presente".

O absenteísmo chamado tipo I (pela falta ao trabalho) é representado pela falta pura e simples do empregado ao trabalho, sendo de fácil mensuração e custo calculado. Leva à perda de produção das horas não trabalhadas.

O absenteísmo tipo II (de corpo presente) é aquele em que, apesar de não faltar ao trabalho, o trabalhador não desenvolve seu melhor desempenho, levando à diminuição na sua produtividade. Isso ocorre devido ao fato de o trabalhador apresentar algum problema de saúde.

Quick \& Lapertosa (1982) dividem o absenteísmo tipo I em cinco classes, cada uma merecendo tratamento e considerações diferentes:

1. Absenteísmo voluntário: é a ausência voluntária do trabalho por razões particulares, não justificadas por doença e sem amparo legal.

2. Absenteísmo compulsório: é o impedimento ao trabalho mesmo que o trabalhador não deseje, por suspensão imposta pelo patrão, por prisão ou outro impedimento que não lhe permita chegar ao local do trabalho.

\section{Objetivos}

O objetivo do trabalho foi analisar o absenteísmo odontológico e médico nos âmbitos público e privado e verificar a interferência de
3. Absenteísmo legal: compreende aquelas faltas ao serviço amparadas por lei, como licença maternidade, nojo, gala, doação de sangue, serviço militar etc. São as chamadas faltas justificadas.

4. Absenteísmo por patologia profissional: Compreende as ausências por doenças profissionais (LER/DORT) ou ausências por acidente de trabalho (infortúnios profissionais).

5. Absenteísmo por doença: inclui todas as ausências por doença ou procedimento médico.

O absenteísmo-doença traz, tanto nos países em desenvolvimento, como nos considerados altamente industrializados, além de um aumento direto dos custos pela concessão de auxílio-doença e um aumento indireto nos custos pela diminuição da produtividade e da eficiência, um maior desperdício e aumento dos problemas administrativos com sucessivas substituições dos faltosos (Diacov \& Lima, 1988).

Os atestados médicos e odontológicos são os únicos instrumentos legais que servem para abonar as faltas ao trabalho por motivo de doença e assegurar o pagamento dos respectivos salários, desde que apresentem a codificação da enfermidade - CID (Nogueira \& Laurenti, 1975), conforme especificado por Acórdão do Tribunal Superior do Trabalho (TST) no dissídio da categoria e estatuto municipal (Araçatuba, 2002; Carrion, 1994; Sime, 2000).

Sempre que possível, é necessário conhecer como se distribui o absenteísmo conforme a idade, o sexo e a ocupação ou cargo dos trabalhadores. A disponibilidade dessas informações é muito importante a fim de facilitar a tomada de decisões das distintas estratégias para sua prevenção (Forssman, 1956; Muñoz, 1997).

Existem diferentes fórmulas para o cálculo do absenteísmo, podendo o mesmo ser realizado através da obtenção de, no mínimo, três índices essenciais: gravidade, freqüência e duração média dos períodos (Quick \& Lapertosa, 1982).

fatores ligados à própria empresa, como regime empregatício, e ao trabalhador, como sexo, idade e função na sua ocorrência. 


\section{Métodos}

A população deste estudo constituiu-se de todos os atestados odontológicos e médicos e declarações de comparecimento, devidamente homologados, emitidos no período de janeiro a junho de 2002 e que deram entrada no departamento pessoal da Prefeitura Municipal de Araçatuba e no da Indústria Color Visão - Indústria Acrílica, cuja finalidade era abonar faltas no serviço de até 15 dias de duração.

Realizou-se a pesquisa nestas duas empresas por serem de grande porte - acima de 500 empregados (Sebrae, 2002) e pelo fato de os diretores/responsáveis consentirem no acesso aos atestados e listagens dos trabalhadores.

\section{Resultados e Discussão}

A carga horária na empresa pública variou de 20, 24, 30, 36 e 40 horas semanais, conforme a função e a atribuição do trabalhador. A prefeitura municipal apresenta-se dividida em secretarias com funcionários em várias funções ligadas à administração pública e que se enquadram, de acordo com a NR 4 (IOB, 1993), nos Graus de Risco de 1 a 3. Já na empresa privada, são trabalhadas 44 horas semanais, independentemente da função do trabalhador. A indústria fabrica máquinas de lavar roupas e móveis com predominância de metal, enquadrando-se no Grau de Risco 3 (IOB, 1993).

No período pesquisado, a empresa pública apresentava 3490 funcionários, sendo 2034 do sexo feminino e 1456 do sexo masculino. A empresa privada apresentava no total 656 empregados, sendo 97 do sexo feminino e 559 do sexo masculino. No total, foram analisados, no período de janeiro a junho de 2002, nas empresas pública (prefeitura) e privada (indústria), 1642 atestados, sendo 64
Destes atestados obtiveram-se dados referentes à data, à duração e ao motivo da falta, baseado no Código Internacional de Doenças - CID-10 (OMS, 1997). Das listagens dos trabalhadores, coletaram-se dados como sexo, idade e função na empresa.

Processaram-se os dados coletados utilizando o programa Epi 2000, v.1.1., considerando-se significativos com $\mathrm{p}<0,0001$.

Para o cálculo do absenteísmo, obtiveramse os índices indicados pela Sociedade Internacional de Saúde Ocupacional (freqüência e gravidade) e a duração média do afastamento pela sua importância (Quick \& Lapertosa, 1982).

odontológicos e 1578 médicos. Do total de atestados, 1311 deram entrada na prefeitura e 331 na indústria.

A relação entre o número de dias perdidos e o número de empregados - Índice de gravidade (Quick \& Çapertosa, 1982) - na empresa pública foi de 1,43 e na empresa privada, de 1,07 , o que significa que a duração das faltas (período de afastamento) no serviço público foi maior. A relação entre o número de períodos de afastamento (ausências) e o número de empregados Índice de freqüência (Quick \& Lapertosa, 1982) - na empresa pública foi de 0,38 e na empresa privada, de 0,50 , o que significa que houve um maior número de atestados (faltas) no serviço privado proporcionalmente ao número de empregados. A relação entre o número de dias perdidos e o número de períodos de afastamento - Duração média das ausências (Quick \& Lapertosa, 1982) na empresa pública foi de 3,82 e na empresa privada, de 2,13.

Tabela I Índices de gravidade, frequiência e duração média das ausências nas empresas pública e privada, Araçatuba, 2002

\begin{tabular}{cccc}
\hline Empresa & Número de dias perdidos & Número de empregados & Índice de gravidade \\
\hline Pública & 5002 & 3490 & 1,43 \\
Privada & 704 & 656 & 1,07 \\
\hline & & & \\
\hline Empresa & Períodos de afastamento & Número de empregados & Índice de frequência \\
\hline Pública & 1311 & 3490 & 0,38 \\
Privada & 331 & 656 & 0,50 \\
\hline & & & \\
\hline Empresa & Número de dias perdidos & Períodos de afastamento & Duração média das ausências \\
\hline Pública & 5002 & 1311 & 3,82 \\
Privada & 704 & 331 & 2,13 \\
\hline
\end{tabular}


Quick \& Lapertosa (1982), no estudo em uma usina siderúrgica e metalúrgica de Belo Horizonte, constataram índice de gravidade de 1,48, índice de freqüência de 0,42 e duração média das ausências de 3,52 faltas, considerando o mesmo período do estudo (6 meses). Danatro (1997) verificou numa instituição pública de Montevideo, no Uruguai, no período de um ano, em relação ao absenteísmo médico, índice de freqüência de 1,08, de gravidade de 6,84 e duração média das faltas de 6,28 dias.

A proporção de absenteísmo foi maior na empresa privada $(50,46 \%)$ que na pública $(37,56 \%)$, estatisticamente significativo através do teste de proporção, com qui-quad =
38,38 e p valor $<0,0001$, cujo regime empregatício é regido pela Consolidação das Leis do Trabalho (CLT). Apesar do maior risco do empregado perder o emprego nesse tipo de regime em comparação ao regime estatutário, o que parece que prevaleceu foi a possibilidade do empregado público estatutário ter como direito as faltas abonadas (Araçatuba, 2002) ,podendo o mesmo usar o dia abonado para tratar de sua saúde, evitando levar um atestado médico ou odontológico à empresa.

Os atestados odontológicos tiveram pouco peso sobre o total de atestados, tanto na empresa pública $(3,3 \%)$, quanto na privada $(6,3 \%)$, o que concorda com os achados de Rocha (1981) e Cartaxo (1982).

Tabela 2 Número e porcentagem dos atestados segundo o tipo nas empresas pública e privada, Araçatuba, 2002

\begin{tabular}{ccc}
\hline Empresa & Atestado odontológico & Atestado médico \\
\hline Pública & $43(3,3 \%)$ & $1268(96,7 \%)$ \\
Privada & $21(6,3 \%)$ & $310(93,7 \%)$ \\
\hline
\end{tabular}

Isso não deve significar que a saúde bucal dos trabalhadores esteja em perfeitas condições, pois Medeiros (1970) observou em um levantamento epidemiológico, realizado com 799 operários de São Paulo, que 52,82\% da população estudada apresentavam um ou mais dentes com extração indicada e 89,74\% dos indivíduos tinham um ou mais dentes cariados.

A ausência do Código Internacional de Doenças (OMS, 1997) na quase totalidade dos atestados odontológicos pode ser a razão para este pequeno peso, pois para o abono das faltas ao trabalho por motivo de doença e pagamento dos respectivos salários é necessário, segundo Acórdão do TST, dissídio da categoria e, também pelo estatuto, a codificação de enfermidade (Araçatuba, 2002; Carrion, 1994; Sime, 2000). Saliba-Garbin et al. (2000) verificaram em seu trabalho que $64,4 \%$ dos dentistas indicavam no atestado o ato praticado, o que sugeriria o desconhecimento do segredo profissional, ou do CID, que foi estabelecido para que os profissionais da área de saúde pudessem se comunicar sem descrever o tratamento realizado, não quebrando, assim, o sigilo profissional.
O capítulo XI do CID (OMS, 1997) agrupa todas as doenças do aparelho digestivo (K00K93), sendo os agrupamentos "K00-K14" referentes às doenças da cavidade oral, das glândulas salivares e dos maxilares. Esses são os agrupamentos de responsabilidade do cirurgião-dentista. Não existe a possibilidade da utilização de um CID médico para designar uma doença odontológica.

Acreditamos que muitas empresas não acatam a lei 6.215 que possibilita ao cirurgiãodentista "atestar no setor de sua atividade profissional, estados mórbidos e outros, inclusive para justificação de faltas no emprego" (Diacov \& Lima, 1988), apenas aceitando o atestado odontológico no caso de urgências, como extrações e abscessos. O empregado pode até conhecer este direito, mas aceita, sem reclamar, a recusa do atestado pela possibilidade de perder o emprego ou de ter sua remuneração descontada.

Houve uma predominância do sexo feminino tanto na empresa pública $(40,2 \%)$, quanto na empresa privada $(55,7 \%)$, concordando com os achados de Cartaxo (1982), Diacov \& Lima (1988) e Danatro (1997).

Tabela 3 Porcentagem de atestados odontológicos e médicos quanto ao sexo, nas empresas pública e privada, Araçatuba, 2002

\begin{tabular}{|c|c|c|c|c|}
\hline & \multicolumn{2}{|c|}{ Empresa pública } & \multicolumn{2}{|c|}{ Empresa privada } \\
\hline & Sexo feminino & Sexo masculino & Sexo feminino & Sexo masculino \\
\hline Total de empregados & 2034 & 1456 & 97 & 559 \\
\hline Atestados & 817 & 494 & 54 & 277 \\
\hline Porcentagem & 40,2 & 33,9 & 55,7 & 49,5 \\
\hline
\end{tabular}


Existe um consenso de que os trabalhadores do sexo feminino apresentam absenteísmo por doença maior que os do sexo masculino, em especial as ausências causadas por ginecopatias (Nogueira \& Laurenti, 1975). Apesar disso, Nogueira \& Laurenti (1975) concluíram em seu estudo que as ginecopatias são causas pouco importantes de absenteísmo nas mulheres, o que concorda com nosso trabalho, que mostrou serem essas causas somente a quinta colocada em número de ocorrências. Para Gomes (1986), a mulher apresenta uma sobrecarga de trabalho causada pelo trabalho dentro e fora do lar e pelas preocupações com a família, embora Cartaxo (1982) tenha verificado em seu estudo um maior número de faltas entre mulheres solteiras, geralmente isentas das atividades dentro do lar e das preocupações com a família.

Dividiu-se a população estudada em faixas etárias de 10 anos, sendo que no serviço público a faixa que mais faltou foi de 30 a 39 anos. No serviço privado, foi a de 20 a 29 anos, o que concorda com o achado por Cartaxo (1982) no seu estudo.

Tabela 4 Número e porcentagem dos atestados odontológicos e médicos segundo a faixa etária nas empresas pública e privada, Araçatuba, 2002

\begin{tabular}{lcc}
\hline $\begin{array}{l}\text { Faixa etária } \\
\text { (empresa pública) }\end{array}$ & $\begin{array}{c}\text { Número de atestados } \\
\text { (empresa privada) }\end{array}$ & Número de atestados \\
\hline Menor de 20 anos & $10(0,8 \%)$ & $30(9,1 \%)$ \\
de 20 a 29 anos & $270(20,6 \%)$ & $197(59,5 \%)$ \\
de 30 a 39 anos & $432(33,0 \%)$ & $53(16,0 \%)$ \\
de 40 a 49 anos & $394(30,1 \%)$ & $46(13,9 \%)$ \\
de 50 a 59 anos & $176(13,4 \%)$ & $5(1,5 \%)$ \\
60 ou mais & $29(2,2 \%)$ & 0 \\
\hline
\end{tabular}

Isso pode ser explicado devido ao fato de os trabalhadores da indústria apresentarem idade menor quando comparados aos trabalhadores da prefeitura. Danatro (1997) verificou maior ocorrência de faltas na faixa etária de 35 a 44 anos de idade.

A doença que mais levou ao afastamento do trabalhador tanto na empresa pública, quanto na privada foi a dorsalgia, cujo CID é o M54 (OMS, 1997), podendo estar ligada à má postura.

A maior parte dos afastamentos por razões odontológicas e médicas tiveram a duração de 1 dia, sendo que as razões odontológicas levaram o trabalhador a afastar-se do labor por até no máximo 5 dias.

Tabela 5 Duração do afastamento segundo o tipo de atestado, Araçatuba, 2002

\begin{tabular}{ccc}
\hline Duração do afastamento & Atestado odontológico & Atestado médico \\
\hline 1 dia & 52 & 720 \\
2 dias & 5 & 293 \\
3 dias & 3 & 128 \\
4 dias & 2 & 82 \\
5 dias & 2 & 65 \\
6 dias & 0 & 19 \\
7 dias & 0 & 57 \\
8 dias & 0 & 28 \\
9 dias & 0 & 9 \\
10 dias & 0 & 34 \\
11 dias & 0 & 3 \\
12 dias & 0 & 6 \\
13 dias & 0 & 3 \\
14 dias & 0 & 6 \\
15 dias & 0 & 125 \\
\hline
\end{tabular}

Deve ser notado que quase $46 \%$ das licenças médicas são de 1 dia, tempo geralmente insuficiente para cura de qualquer doença. Pode ser dia perdido para um simples exame complementar ou para outros procedimentos médicos, como também ser "doença provocada”. A licença médica muitas vezes é utilizada pelo trabalhador como uma forma de agressão à chefia e à instituição; também representa uma válvula 
de escape de tensões sociais, econômicas e psicológicas. É um ponto crítico a ser atacado na luta contra o absenteísmo. Um menor número de períodos (ausências) com maior número de dias perdidos indica sempre doença real (Quick \& Lapertosa, 1982; Valtorta, Sidi \& Bianchi, 1985).

A função em que ocorreu o maior número de faltas no serviço privado, proporcionalmente ao número de empregados, foi a de operador de injetora de plástico, que é uma função ligada à linha de produção. Já no serviço público foi a

\section{Conclusões}

O absenteísmo nas empresas pública e privada deu-se mais por razões médicas, quando comparado com as razões odontológicas, devido ao maior número de faltas e à maior duração das mesmas, levando aos problemas decorrentes da falta do trabalhador em seu serviço. O tipo de regime empregatício e os fatores ligados ao trabalhador, como sexo, idade e função, influenciaram no absenteísmo ao trabalho.

Acreditamos que o trabalhador estatutário ausentou-se menos no trabalho por apresentar como um dos seus direitos a "falta abonada", podendo utilizá-la para tratar de sua saúde.

As razões médicas causaram o afastamento do trabalhador por 5604 dias, enquanto as razões odontológicas levaram o trabalhador a ausentar-se do labor por 89 dias no período de estudo (6 meses). função de auxiliar de serviços gerais 1. Em ambas as empresas, o maior número de faltas ocorreu nos serviços não burocráticos (administrativos). Cartaxo (1982) verificou em seu estudo que a maioria das faltas eram de trabalhadores menos qualificados e com menor salário. Pode-se acreditar que os trabalhadores que ganham um salário maior faltem menos porque estão satisfeitos com o seu salário ou se nutrem melhor adoecendo menos. Danatro (1997) verificou em uma instituição pública maior ocorrência de faltas nos serviços administrativos.

Importante deve ser a orientação ao profissional cirurgião-dentista da necessidade da Codificação Internacional de Doenças no atestado odontológico com a finalidade de abonar a falta do trabalhador ao serviço.

Novos estudos precisarão ser realizados, pois, levantada à distribuição do absenteísmo conforme idade, sexo e ocupação ou cargo dos trabalhadores, ou seja, realizada uma abordagem quantitativa do problema, deverá ser feita uma análise qualitativa dos problemas especificamente a estes trabalhadores a fim de prevenir sua ocorrência.

Haverá a necessidade de um trabalho multiprofissional na empresa devido à complexidade do absenteísmo e por estarem envolvidos muito mais fatores que a simples doença.

\section{Agradecimentos}

Ao diretor e aos funcionários da Secretaria de Governo e Gestão Estratégica da Prefeitura Municipal de Araçatuba e da indústria Color-Visão do Brasil - Indústria Acrílica Ltda., que permitiram a realização deste trabalho.

\section{Referências Bibliográficas}

ARAÇATUBA (Prefeitura). Estatuto. Lei $\mathrm{n}^{\mathrm{o}}$ 3774. Disponível em <www.aracatuba. sp.gov.br> Acesso em outubro de 2002.

CARRION, V. Nova jurisprudência em direito do trabalho. São Paulo: Revista dos Tribunais, 1994. 702p.

CARTAXO, R. M. S. Absenteísmo em empresas industriais de Campina Grande-Paraíba: um estudo de suas causas. 1982. Dissertação (Mestrado em Ciências da Saúde) Faculdade de Odontologia, Universidade Federal do Rio Grande do Norte.

DANATRO, D. Absentismo laboral de causa médica en una institución pública. Revista
Médica del Uruguay. Montevideo, 13(2): 101-109, 1997.

DIACOV, N.; LIMA, J. R. S. Absenteísmo odontológico. Revista de Odontologia da UNESP. São Paulo, 17(1/2): 183-189, 1988.

FORSSMAN, S. El absentismo en la industria. Bol. Oficina Sanitaria Panam. Washington, 41(6): 526-530, 1956.

GOMES, D. L. A mulher, seu trabalho e as implicações em saúde. Revista Paulista de Enfermagem. São Paulo, 6(2): 91-96, 1986.

IOB. Segurança e saúde no trabalho. 3 ed. São Paulo: Gráfica Editora, 1993. 314p. 
JORGE, A. L. Motivos que levam os trabalhadores de enfermagem ao absenteísmo. Acta Paulista de Enfermagem. São Paulo, 8(1): 39-46, 1995.

MEDEIROS, E. P. G. Necessidades de tratamento odontológico em operários de São Paulo. O Incisivo. São Paulo, 9(1): 13-21, 1970.

MIDORIKAWA, E. T. A odontologia em saúde do trabalhador como uma nova especialidade profissional: definição do campo de atuação e funções do cirurgião-dentista na equipe de saúde do trabalhador. 2000. Dissertação (Mestrado em Odontologia) Faculdade de Odontologia, Universidade de São Paulo.

MUÑOZ, M. M. Absentismo laboral. In: BENAVIDES, F. G., RUIZ-FRUTOS, C., GARCÍA GARCÍA, A. M. Salud laboral: conceptos y técnicas para la prevención de riesgos laborales. Barcelona: Masson, 1997.

NOGUEIRA, D. P.; LAURENTI, R. Absenteísmo por doença em mulheres. Revista de Saúde Pública. São Paulo, 9(3): 393-399, 1975.

ORGANIZAÇÃO MUNDIAL DE SAÚDE. CID10: classificação estatística internacional de doenças e problemas relacionados à saúde. 5 ed. São Paulo: Edusp, 1997. 1191p.

PINTO, V. G. Saúde Bucal: odontologia social e preventiva. 3 ed. São Paulo: Santos, 1992. 415p.
QUICK, T. C.; LAPERTOSA, J. B. Análise do absentismo em usina siderúrgica. Revista Brasileira de Saúde Ocupacional. São Paulo, 10(40): 62-67, 1982.

ROCHA, J. A. D. Absenteísmo ao trabalho por doença e a implicação da saúde bucal como um dos seus fatores numa indústria metalúrgica da cidade de Canoas. 1981. Dissertação (Mestrado em Odontologia Social) Centro de Pesquisa em Odontologia Social, Faculdade de Odontologia, Universidade Federal do Rio Grande do Norte.

SALIBA-GARBIN, C. A.; MOIMAZ, S. A. S.; SALIBA, T. A.; GARBIN, A. J. I. O cirurgiãodentista e a emissão de atestados odontológicos. Odontologia Social. São Paulo, 2(1/ 2): 89-92, 2000.

SEBRAE. O Sistema Sebrae: histórico. Disponível em <http://www.electus. com.br/sebraece/sbr1.htm > Acesso em setembro de 2002.

SIME. Convenção coletiva de trabalho. Araçatuba, 2000. 23p.

VALTORTA, A.; SIDI, E.; BIANCHI, S. C. Estudo do absenteísmo médico num hospital de grande porte. Revista Brasileira de Saúde Ocupacional. São Paulo, 13(51): 55-61, 1985. 